\title{
A High-Speed Delta-Sigma Modulator with Relaxed DEM Timing Requirement
}

\author{
Sunwoo Kwon and Un-Ku Moon \\ Electrical Engineering and Computer Science \\ Oregon State University, Corvallis, OR
}

\begin{abstract}
This paper presents a high-speed digital feedforward Delta-Sigma Modulator which relaxes timing requirement for the Dynamic Element Matching (DEM) algorithm. By making the main Digital to Analog Converter (DAC) process a small part of the input signal, the distortion from the DAC is suppressed. The proposed method allows eliminating the DEM circuitry in the critical data path for high-speed applications, thus achieving high-resolution without augmenting considerable silicon area. Analysis and simulation results verify the effectiveness of the proposed method.
\end{abstract}

\section{INTRODUCTION}

A Delta-Sigma Modulator $(\Delta \Sigma \mathrm{M})$ is a popular solution for high-resolution and low-power wideband telecommunication systems. This is due to the fact that low-accuracy circuit components can be utilized with less design effort via the trade-off between speed and resolution, compared to a Nyquist-rate data converter. Traditionally, $\Delta \Sigma \mathrm{Ms}$ with a single-bit quantizer have been implemented in fully discretetime topologies such as switched capacitor (SC) $\Delta \Sigma \mathrm{Ms}$. They can exploit good capacitor matching properties and inherently linear behavior of the single-bit DAC, and consequently they achieve highly accurate transfer functions and highly linear analog-to-digital conversion. However, the performance of a single-bit SC $\Delta \Sigma \mathrm{M}$ is limited by $\mathrm{kT} / \mathrm{C}$ noise due to the inherent sampling nature at the input of the SC $\Delta \Sigma \mathrm{M}$. Furthermore, the Operational Amplifiers (OpAmps) in the modulator should be fast enough to ensure a proper settling within a half clock period [1].

To obtain further performance improvement without the added stability problem and power consumption, a single-bit quantizer may be replaced with a multi-bit quantizer [1]. However, the multi-bit $\Delta \Sigma \mathrm{M}$ is prone to the Digital to Analog Converter (DAC) nonlinearity due to lithographic errors during fabrication that ultimately limit the Signal-toNoise-and-Distortion Ratio (SNDR) to around 10 bits [1], [2] depending on the size of the DAC elements. So as to achieve better linearity performance, one can utilize a DAC linearization techniques such as the Dynamic Element

This work is supported by Samsung Electronics.

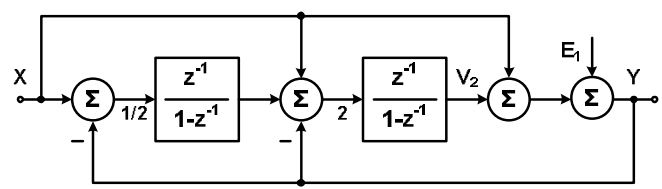

Figure 1. Conventional $2^{\text {nd }}$ order wideband $\Delta \Sigma \mathrm{M}$

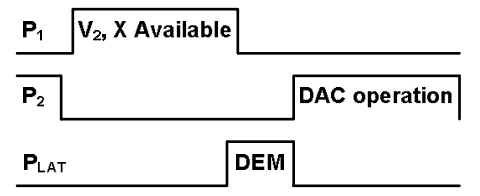

Figure 2. Timing diagram for the structure shown in Fig. 1

Matching (DEM) techniques [3], [4], component sorting [5], or component sizing method [6]. Both the sorting and sizing methods, however, increase silicon area due to the fact that the sorting method requires the registers to contain comparison results and demands complex routing, and that the sizing method calls for larger components as the required resolution increases higher. On the other hand, the DEM method employs an averaging scheme, so that all of the elements in the DAC are equally used. As a result, silicon augmentation is minimal and the DEM technique effectively linearizes the nonlinear feedback DAC.

The DEM technique is not free from limitations, however. It needs considerable amount of time to execute the algorithm and is inappropriate in high frequency operation since it should be performed in the time slot (non-overlap time of clock phases) between quantization and DAC operations. This timing problem is more critical in a highspeed continuous-time $\Delta \Sigma \mathrm{M}$ as its operating frequency goes beyond half $\mathrm{GHz}$ [6]. Figs. 1 and 2 illustrate a conventional wideband $\Delta \Sigma \mathrm{M} \mathrm{[7]}$ and a timing diagram related to the DEM operation, respectively. Ideally, the DEM algorithm can be processed before the DAC operation completes, but it burdens the first stage OpAmp in the modulator. In other words, the first OpAmp should be much faster to guarantee a 


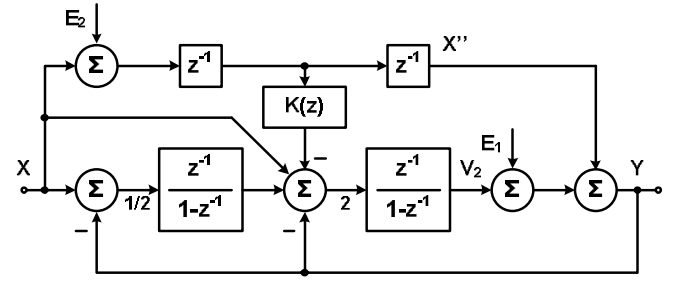

Figure 3. $2^{\text {nd }}$ order wideband $\Delta \Sigma \mathrm{M}$ with digital feed-forward

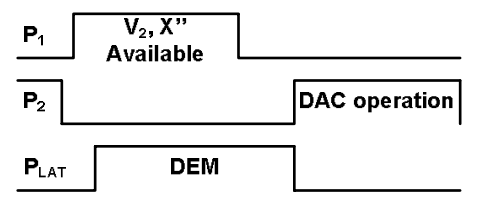

Figure 4. Timing diagram for the structure shown in Fig. 3

proper settling during the DAC operation.

Thus, it is necessary to develop a technique that allows the $\Delta \Sigma \mathrm{M}$ to operate at a higher clocking frequency without having to face a severe DAC nonlinearity problem. This paper is organized as follows. In Section II, detailed problem with the DEM in high-speed applications and operational principles are discussed. Various simulation results are presented in Section III. Finally, conclusions are given in Section IV.

\section{DEM IN High-SPEED APPLICATIONS}

\section{A. High-Speed $\triangle \Sigma M$}

As the operating clock frequency increases, the OpAmps in the modulator become more power-hungry and so does the quantizer. To relax power consumption requirement, one can use the feed-forward technique in the analog [7], [8] or in the digital domain [9], [10]. As a result, the OpAmps are virtually independent from the input signal and process only the quantization noise.

Reference [7] and [8], however, have a tight timing specification for operating the DEM algorithm as shown in Fig. 2: The input signal $X$ should be summed with the output of the $2^{\text {nd }}$ integrator $V_{2}$, and the summed result should be resolved during phase $1\left(P_{l}\right)$ to have a proper settling or charge distribution, depending on the type of analog adder used. Otherwise, the input signal is delayed and the signal transfer function of the modulator is not unity anymore.

On the other hand, [9] has relatively a relaxed timing for the DEM; since $V_{2}$ is already available at the end of phase 2 $\left(P_{2}\right)$ and the delayed input signal is always ready for the entire clock cycle, the main quantizer, $Q_{1}$, can be activated at the beginning of $P_{l}$. Figs. 3 and 4 show the block diagram and the timing diagram of [10]. Despite the relaxed requirement, at a very high clocking frequency, it may still be difficult to perform a DEM algorithm in the main path which contains the main quantizer, $\mathrm{Q}_{1}$.

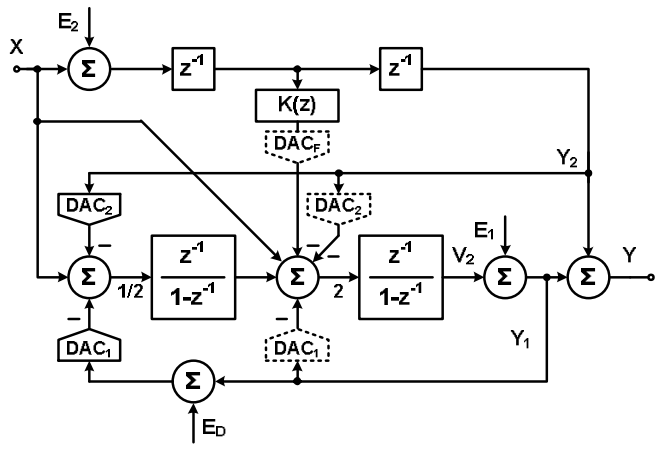

Figure 5. Redrawn block diagram

\section{B. Relaxing the DEM timing requirement}

A closer examination of the topology in Fig. 3 gives a possible solution to relax the DEM timing problem, and Fig. 5 shows a redrawn block diagram which is functionally equal to the one shown in Fig. 3.

Fig. 5 shows that the loop filter receives the input signal, $X-Y_{2}$. Deriving the system transfer function shows that

$$
\begin{aligned}
& Y_{1, I D E A L}=2\left(1-z^{-2}\right) X+\left(1-z^{-1}\right)^{2} E_{1}-z^{-2} E_{2} w / o E_{D} \\
& Y_{1, N O N I D E A L}=Y_{1, I D E A L}+z^{-2} E_{D} \text { with } E_{D} \\
& \left.E_{2}=V_{R E F} / \text { (Number of quantization levels of } Q_{2}\right)
\end{aligned}
$$

where, $Y_{1}$ stands for the output of the main quantizer, $X$ for the input signal, $E_{1}$ for the main quantization noise, $E_{2}$ for the auxiliary quantization noise, and $V_{R E F}$ for the reference voltage. The main DAC nonlinearity, $E_{D}$, however, is not shaped by the loop filter as shown in eq. (2) unlike the multistage noise shaping structure whose second stage is fed into a high-pass filter to cancel out the quantization noise of the first stage [1]. The distortion error of the main DAC shown in Fig. 5, however, is smaller than the DAC distortion error generated from the structure shown in Fig. 1. It is due to the fact that $Q_{1}$ ideally processes the quantization error of the auxiliary quantizer, $E_{2}$. As a result, $\mathrm{DAC}_{1}$ creates negligible amount of distortion as the number of bits of $Q_{2}$ increases.

\section{Quantization Levels of the Auxiliary Quantizer}

As mentioned above, increasing the number of quantization levels of $Q_{2}$ helps to minimize the nonlinearity of $\mathrm{DAC}_{1} . E_{2}$, however, cannot be infinitely small, since increasing the number of quantization levels of $Q_{2}$ requires more hardware, the implementation of which is typically a flash analog to digital converter (ADC) where the number of comparators would increase dramatically with the increase in the number of bits.

Another way to improve the resolution of $Q_{2}$ would be to replace the flash $\mathrm{ADC}$ with a two-step $\mathrm{ADC}$ or a pipeline $\mathrm{ADC}$, so that the number of comparators would be reduced. This substitution does not consume a considerable amount of power since any error such as the OpAmp settling error and 
the $\mathrm{kT} / \mathrm{C}$ noise in the digital feed-forward path is cancelled out or at least shaped by the loop filter. This remains true even when there are path mismatches between the digital and analog paths [9], [10]. The number of stages of a pipeline $\mathrm{ADC}$, however, is bound by the available time for the DEM operation in the digital feed-forward path.

\section{DEM in the digital feed-forward path}

In spite of the fact that the necessity of employing a $\mathrm{DEM}$ technique between the main quantizer and $\mathrm{DAC}$ is removed, the auxiliary $\mathrm{DAC}, \mathrm{DAC}_{2}$, still needs a DEM technique to linearize the DAC elements. This requirement, however, is no longer critical since it can use a full clock period or even more time (as much as the modulator is allowed to have). For example, in case of the $2^{\text {nd }}$ order modulator, it is acceptable for the DEM operation to take two full clock periods. Since the output of the noise cancellation filter, $K(z)$, is injected into the input of the second integrator, it is shaped by a $1^{\text {st }}$ order. Hence, the noise cancellation DAC does not require linearization/DEM.

\section{Simulation ReSUlts}

To demonstrate the validity of removing DAC linearization (DEM) in the main DAC, three $3^{\text {rd }}$ order $\Delta \Sigma \mathrm{Ms}$ are simulated using the MATLAB/Simulink ${ }^{\circledR}$ model [11], [12] under various conditions: one analog feed-forward $\Delta \Sigma \mathrm{M}$ [7] as a reference and two digital feed-forward $\Delta \Sigma \mathrm{Ms}$ as illustrated in Fig. 6, one with a 4 b auxiliary quantizer and the other with a 5 b auxiliary quantizer. All simulations are carried out assuming that $\mathrm{DAC}_{2}$ is linearized with the help of the DEM technique [10]. Simulation parameters are summarized in Table I.

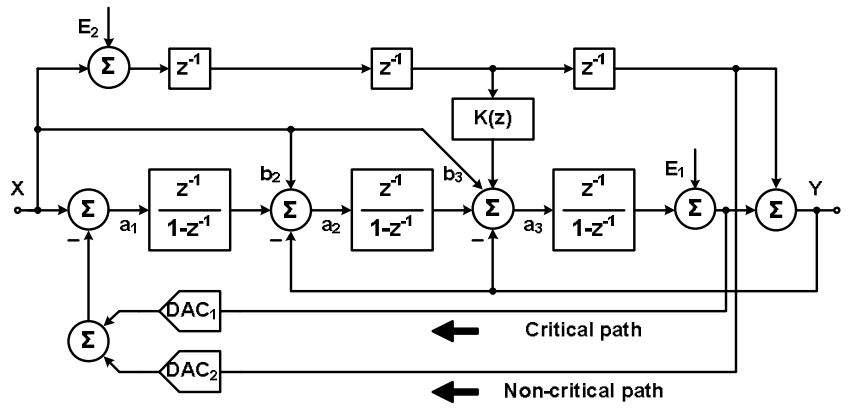

Figure 6. $3^{\text {rd }}$ order $\Delta \Sigma \mathrm{M}$ without linearizing the main $\mathrm{DAC}, \mathrm{DAC}_{1}$

TABLE I. SIMULATION PARAMETERS

\begin{tabular}{|l|c|}
\hline \multicolumn{1}{|c|}{ Parameter } & Value \\
\hline Over sampling ratio & 16 \\
\hline Signal bandwidth $^{\mathrm{a}}$ & 0.031 \\
\hline Input signal amplitude & $-3 \mathrm{dBFS}$ \\
\hline Number of points for FFT & 65536 \\
\hline Main quantizer resolution & 4-bit \\
\hline
\end{tabular}

a. Normalized to the sampling frequency

\section{A. Main DAC nonlinearity}

As expected from eqs. (2) and (3), the nonlinearity generated from $\mathrm{DAC}_{1}$ is minimized as the magnitude of quantization error, $E_{2}$, decreases. Fig. 7 shows SNDR versus $\mathrm{DAC}_{1}$ bit accuracy. It can be found that $\mathrm{DAC}_{1}$ becomes insensitive to DAC nonlinearity, as the quantization error of $Q_{2}$ decreases. This, however, does not completely eliminate the harmonics. Fig. 8 shows power spectral density for 3 different modulators with the same $7 \mathrm{~b}$ main DAC accuracy. SNDR degradation due to the nonlinear DAC, however, is not significant. The degradations are $3 \mathrm{~dB}$ and $0.1 \mathrm{~dB}$ for $4 \mathrm{~b}$ and $5 \mathrm{~b}$ auxiliary quantizer, respectively. Furthermore, the loss is less critical when other nonideal effects, such as kT/C noise and settling error, are considered. Table II summarizes the simulation results for each of these three cases with an additional baseline case involving ideal linear main DAC.

It is notable that the conventional modulator shows severe SNDR degradation. This is due to the fact that there is only one DAC which processes the whole input signal. In such conventional design, it is necessary to use a DEM algorithm (under tight/difficult time window allotted for this task) to suppress DAC nonlinearity.

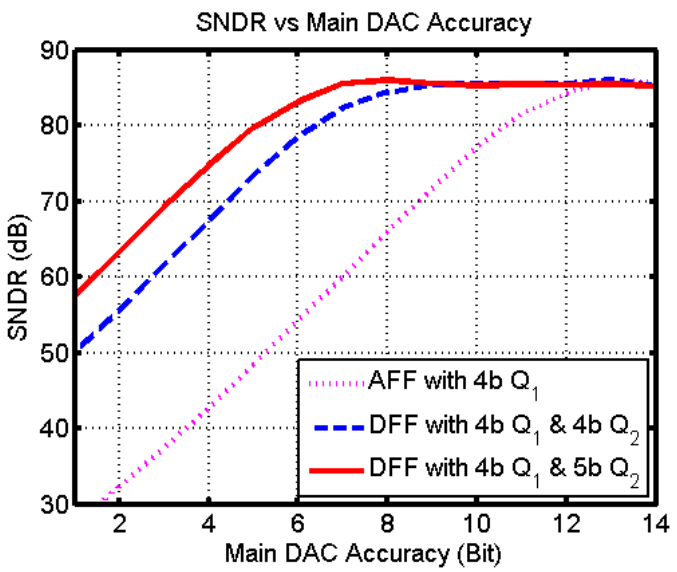

Figure 7. SNDR vs Main DAC accuracy

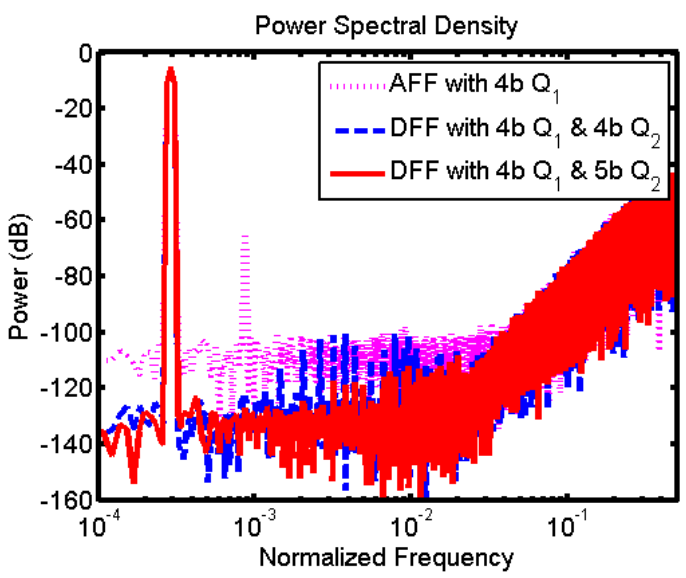

Figure 8. Power spectral density for 3 different cases 
TABLE II. SIMULATION RESULTS

\begin{tabular}{|l|l|}
\hline \multicolumn{1}{|c|}{ Modulator } & SNDR \\
\hline Ideal $3^{\text {rd }}$ order AFF $\Delta \Sigma \mathrm{M}$ & $85.5 \mathrm{~dB}$ \\
\hline $3^{\text {rd }}$ order AFF $\Delta \Sigma \mathrm{M}$ with DAC nonlinearity & $60.0 \mathrm{~dB}$ \\
\hline $\begin{array}{l}3^{\text {rd }} \text { order DFF } \Delta \Sigma \mathrm{M} \text { with DAC nonlinearity } \\
\text { and } 4 \mathrm{~b}_{2}\end{array}$ & $82.4 \mathrm{~dB}$ \\
\hline $\begin{array}{l}3^{\text {rd }} \text { order DFF } \Delta \Sigma \mathrm{M} \text { with DAC nonlinearity } \\
\text { and } 5 \mathrm{~b} \mathrm{Q}_{2}\end{array}$ & $85.4 \mathrm{~dB}$ \\
\hline
\end{tabular}

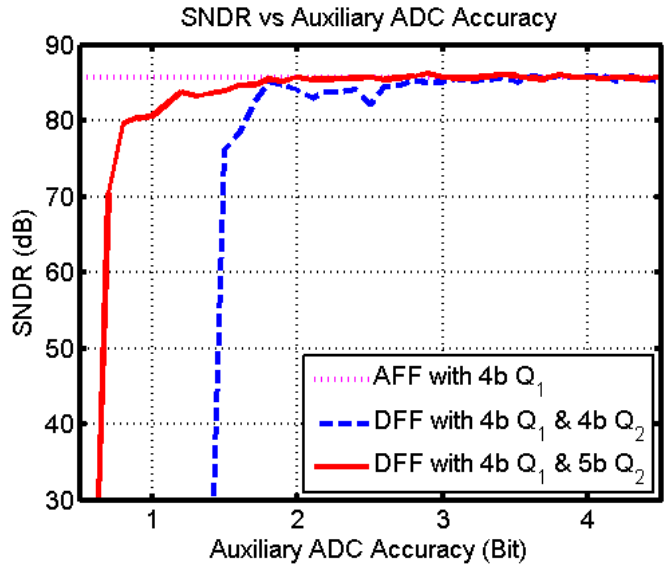

Figure 9. SNDR vs Auxiliary ADC accuracy

\section{B. Auxiliary quantizer nonlinearity}

Nonlinearity generated from the auxiliary quantizer is not detrimental since it is cancelled out at the modulator output as mentioned before. Fig. 9 shows that the modulator is robust to nonlinearity from the auxiliary quantizer but, after a certain point, the modulator performance is undermined due to the available swing limitation of the integrators. This condition, however, is easily avoided by decreasing the quantization error of the auxiliary quantizer.

The conventional modulator does not show any degradation in the figure, because the analog feed-forward path does not utilize an auxiliary quantizer. However, it is important to remember once again that the conventional topology is not suitable for high-speed applications due to the lack of time available for the DEM logic circuitry to work properly.

\section{CONCLUSIONS}

A high-speed $\Delta \Sigma M$ with a relaxed DEM timing requirement has been presented. By eliminating the DEM circuitry in the critical path between the main quantizer and the DAC, the proposed modulator can operate at much higher clocking frequency without increasing the power consumption of integrators. Employing the DEM in the feedforward path becomes an easy task since this path can have more than a full clock delay (specifically two full clock periods in the example shown). Obviating the use of the DEM algorithm in the critical path enables the modulator to minimize the size of the DAC element without degrading modulator's performance, thus economizing silicon area. Furthermore, increasing the number of levels of the auxiliary quantizer may possibly be achieved by replacing the quantizer with a two-step or a pipeline ADC. Naturally this implementation possibility would depend very much on the number of delays in the digital feed-forward path. The presented $\Delta \Sigma \mathrm{M}$ example is suitable for high-speed and highresolution applications.

\section{ACKNOWLEDGMENT}

S. Kwon thanks N. Maghari and R. Gregoire for valuable discussion.

\section{REFERENCES}

[1] S. R. Norsworthy, R. Schreier, and G. C. Temes, Delta-Sigma Data Converters: Theory, Design and Simulation. Piscataway, NJ: IEEE Press, 1997.

[2] A. Hastings, The Art of Analog Layout. Upper Saddle River, NJ: Prentice-Hall, Inc., 2001.

[3] F. Chen and B. H. Leung, "A High Resolution Multibit Sigma-Delta Modulator with Individual Level Averaging," IEEE J. Solid-State Circuits, vol. 30, pp. 453-460, Apr. 1995.

[4] R. T. Baird and T. S. Fiez, "Linearity Enhancement of Multibit $\Delta \Sigma$ $\mathrm{A} / \mathrm{D}$ and $\mathrm{D} / \mathrm{A}$ Converters Using Data Weighted Averaging," IEEE Trans. Circuits Syst. II, vol. 42, pp. 753-762, Dec. 1995.

[5] S. Ray and B. Song, "A 13b Linear 40MS/s Pipelined ADC with SelfConfigured Capacitor Matching," in ISSCC Dig. Tech. Papers, Feb. 2006, pp. 228-229.

[6] G. Mitteregger et al., "A 14b 20mW 640MHz CMOS CT $\Delta \Sigma$ ADC with 20MHz Signal Bandwidth and 12b ENOB," in ISSCC Dig. Tech. Papers, Feb. 2006, pp. 62-63.

[7] R. Gaggl, M. Inversi, and A. Wiesbauer, "A Power Optimized 14-Bit SC $\Delta \Sigma$ Modulator for ADSL CO Applications," in ISSCC Dig. Tech. Papers, Feb. 2004, pp. 82-83.

[8] J. Silva, U. Moon, J. Steensgaard, and G. C. Temes, "Wideband LowDistortion Delta-Sigma ADC Topology," IEE Elec. Letters, vol. 37, pp. 737-738, June 2001.

[9] S. Kwon and F. Maloberti, "Opamp swing reduction in Sigma-Delta Modulator" in Proc. IEEE ISCAS 2004, May 2004, pp. 525-528.

[10] S. Kwon and F. Maloberti, "A $14 \mathrm{~mW}$ Multi-bit $\Delta \Sigma$ Modulator with $82 \mathrm{~dB}$ SNR and $86 \mathrm{~dB}$ DR for ADSL2+," in ISSCC Dig. Tech. Papers, Feb. 2006, pp. 68-69.

[11] P. Malcovati et al., "Behavioral Modeling of Switched-Capacitor Sigma-Delta Modulators," IEEE Trans. Circuits Syst. I, vol. 50, pp. 352-364, Mar. 2003.

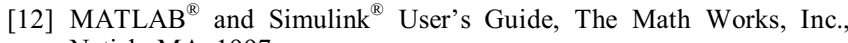
Natick, MA, 1997. 